\title{
Bystander Effect between Zebrafish Embryos in Vivo Induced by High-Dose X-rays
}

\author{
V. W. Y. Choi, ${ }^{\dagger}$ C. Y. P. Ng, A. Kobayashi, ${ }^{\dagger}$ T. Konishi, ${ }^{\ddagger}$ N. Suya, ${ }^{\ddagger}$ T. Ishikawa, ${ }^{\ddagger}$ S. H. Cheng, ${ }^{\S}, \|$ \\ and K. N. Yu ${ }^{\dagger, \|, *}$ \\ ${ }^{\dagger}$ Department of Physics and Materials Science, City University of Hong Kong, Tat Chee Avenue, Kowloon, Hong Kong SAR, \\ Hong Kong \\ ${ }^{\ddagger}$ Department of Technical Support and Development, National Institute of Radiological Sciences, Chiba 263-8555, Japan \\ ${ }^{\S}$ Department of Biology and Chemistry, City University of Hong Kong, Tat Chee Avenue, Kowloon, Hong Kong SAR, Hong Kong \\ "State Key Laboratory in Marine Pollution, City University of Hong Kong, 31 To Yuen Street, Kowloon, Hong Kong SAR, Hong Kong
}

Supporting Information

\begin{abstract}
We employed embryos of the zebrafish, Danio rerio, for our studies on the in vivo bystander effect between embryos irradiated with highdose X-rays and naive unirradiated embryos. The effects on the naive whole embryos were studied through quantification of apoptotic signals at $25 \mathrm{~h}$ post fertilization (hpf) through the terminal dUTP transferase-mediated nick endlabeling (TUNEL) assay followed by counting the stained cells under a microscope. We report data showing that embryos at 5 hpf subjected to a 4-Gy $\mathrm{X}$-ray irradiation could release a stress signal into the medium, which could induce a bystander effect in partnered naive embryos sharing the same medium. We further demonstrated that this bystander effect (induced through partnering) could be successfully suppressed through the addition of the nitric oxide (NO) scavenger 2-(4-carboxyphenyl)-4,4,5,5-tetramethylimidazoline-1oxyl-3-oxide (cPTIO) into the medium but not through the addition of the

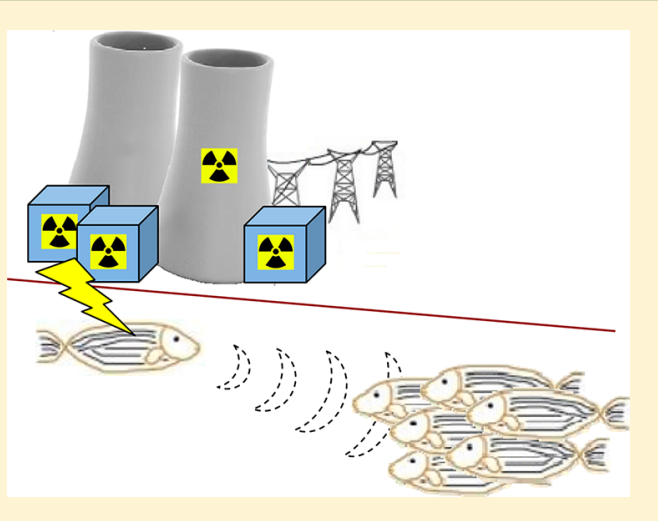
CO liberator tricarbonylchloro(glycinato)ruthenium(II) (CORM-3). This shows that NO was involved in the bystander response between zebrafish embryos induced through X-ray irradiation. We also report data showing that the bystander effect could be successfully induced in naive embryos by introducing them into the irradiated embryo conditioned medium (IECM) alone, i.e., without partnering with the irradiated embryos. The IECM was harvested from the medium that had conditioned the zebrafish embryos irradiated at $5 \mathrm{hpf}$ with 4-Gy X-ray until the irradiated embryos developed into 29 hpf. NO released from the irradiated embryos was unlikely to be involved in the bystander effect induced through the IECM because of the short life of NO. We further revealed that this bystander effect (induced through IECM) was rapidly abolished through diluting the IECM by a factor of $2 \times$ or greater, which agreed with the proposal that the bystander effect was an on/off response with a threshold.
\end{abstract}

\section{INTRODUCTION}

Many radioecological effects of fallouts from nuclear accidents have remained unknown, and it is indeed pertinent to study these in more detail. In nuclear accidents such as that in Fukushima, a high radiation dose of a few Gy can be absorbed by wildlife within a short period of time, for example, a few days. According to the wildlife dose reconstruction for the Fukushima event carried out by Garnier-Laplace et al., ${ }^{1}$ the maximum dose rates for ${ }^{131} \mathrm{I},{ }^{134} \mathrm{Cs}$, and ${ }^{137} \mathrm{Cs}$ was about $2600 \mathrm{mGyd}^{-1}$ for benthic biota - fish, molluscs, and crustaceans. In other words, a dose of 4 Gy could have been achieved within less than $2 \mathrm{~d}$. As such, these high dose levels are still of environmental relevance under extenuating circumstances such as a nuclear accident. In the present work, the radiation-induced bystander effect (RIBE) on the interorganism level induced by a high radiation dose was studied.

RIBE in cells refer to the phenomenon that unirradiated cells respond as if they have been irradiated after having partnered with the irradiated cells or after having been introduced into the medium previously conditioning the irradiated cells. Surinov et al. $^{2}$ reported RIBE between mice and revealed that the bystander signal was transmitted through urine. RIBE has also been shown to occur between fish. Mothersill et al. demonstrated RIBE between fish by showing that X-rayirradiated freshwater rainbow trout (Oncorhynchus mykiss, $W$ ) released bystander signals into the water to induce bystander effects in unirradiated naive partners, which was proved through the increased death of reporter cells induced by the media from explants from cultured tissues of the naive partners. ${ }^{3}$ Mothersill et al. ${ }^{4,5}$ also demonstrated RIBE between zebrafish (Danio rerio) and between Medaka (Oryzias latipes).

Received: March 17, 2013

Revised: May 10, 2013

Accepted: May 13, 2013

Published: May 13, 2013 
Mothersill et al. ${ }^{3}$ put forward a visionary proposal that the RIBE was an evolutionarily conserved effect designed to provide an effective population response. In going along the line of this proposal, our group studied the derived benefit in terms of induction of radioadaptive response (RAR) by communication of radiation-induced bystander signals. ${ }^{6}$ RAR refers to the phenomenon where a small preceding priming dose decreases the biological effectiveness of a subsequent large challenging dose. Olivieri et al. ${ }^{7}$ first reported RAR in peripheral blood lymphocytes, and Cai et al. ${ }^{8}$ and Wang et al. ${ }^{9}$ subsequently exhibited RAR induced between mice. Our group then succeeded to demonstrate that embryos of the zebrafish, Danio rerio, irradiated by low-dose alpha particles released a stress signal into the water to induce RAR in the naive embryos sharing the same water medium with the irradiated embryos. ${ }^{6}$ More recently, our group further studied the derived benefit in terms of induction of hormetic effect by communication of radiation-induced bystander signals. ${ }^{10}$ Hormetic responses are presented as biphasic dose-response relationships characterized by a low-dose stimulation and a high-dose inhibition. ${ }^{11-13}$ Our results showed that, for low alpha-particle dose, the number of apoptotic signals decreased in the irradiated embryos and also in the unirradiated bystander embryos having partnered with the irradiated embryos. These suggested that alphaparticle-irradiated zebrafish embryos could release a stress signal into the water, which could be communicated to unirradiated bystander zebrafish embryos sharing the same water medium to induce a hormetic effect in the bystander embryos. ${ }^{10}$

Most research on RIBE involved low radiation doses ( $\sim 5 \mathrm{mGy}){ }^{14}$ whereas doses as low as 1 cGy could already lead to RIBE. ${ }^{15}$ Nevertheless, RIBE was also induced by high radiation doses. ${ }^{15,16}$ In particular, an X-ray dose of 10 Gy could still induce RIBE. ${ }^{15,16}$ As such, it was also suspected that a high radiation dose (say $4 \mathrm{~Gy}$ ) derived from fallouts from a nuclear accident would be able to induce RIBE on the interorganism level.

In the present work, embryos of the zebrafish, Danio rerio, were employed as the model for studying the stress signal released by an irradiated aquatic organism into the water, which communicated the radiation-induced bystander effect. Its rapid embryonic development has remarkably saved time for the experiments. There have been a growing number of studies using zebrafish or zebrafish embryos as a vertebrate model to study the in vivo response to ionizing radiation. ${ }^{4,17-24}$ Incidentally, Danio rerio has become a preferred vertebrate model for studying human disease in recent years because the zebrafish and human genomes share considerable homology including conservation of most DNA repair-related genes. ${ }^{25}$

The present work first aimed to examine the in vivo bystander effect between partnered zebrafish embryos induced by high-dose $\mathrm{X}$-rays and to assess the corresponding stress communicated between the irradiated and naive embryos through studying changes in the bystander effect after the introduction of the $\mathrm{CO}$ liberator tricarbonylchloro(glycinato)ruthenium(II) (CORM-3) and the nitric oxide (NO) scavenger 2-(4-carboxyphenyl)4,4,5,5-tetramethylimidazoline-1-oxyl-3-oxide (cPTIO) into the water medium. The present work then aimed to characterize the irradiated embryo conditioned medium (IECM) with a view to gather more information on the stress signals released by the irradiated embryos. The capability of the IECM in inducing the bystander effect in naive embryos as well as its abolishment through dilution of the IECM were examined.

\section{MATERIALS AND METHODS}

Experimental Animals. Adult zebrafish (Danio rerio) were kindly provided by RIKEN Brain Science Institute, JAPAN (courtesy Prof. Hitoshi Okamoto). The fish were reared in glass tanks in a laboratory with an ambient temperature of $28{ }^{\circ} \mathrm{C}$ and with a $14 / 10$ h light-dark cycle. Special collectors (ref 26) were lowered onto the bottom of each tank at the time the photoinduced spawning began to collect embryos for a brief period lasting 15 to $30 \mathrm{~min}$ to ensure synchronization of the developmental stage of the collected zebrafish embryos. These collected embryos were rinsed with deionized water and then incubated in a $28.5{ }^{\circ} \mathrm{C}$ incubator to allow continuous development. At 4 hpf, healthy developing embryos were selected and transferred into a Petri dish lined with a layer of agarose gel as the substrate for dechorionation using a pair of sharp forceps under the stereomicroscope.

Exposure Protocols. Our experiments had four parts as explained in parts (A) to (D) in the following. An X-ray generator (TITAN, Shimazu Corporation, Kyoto, Japan) set at $200 \mathrm{kVp}$ and $20 \mathrm{~mA}$ was employed to irradiate the zebrafish embryos, and irradiation was made through a copper and aluminum filter with a thickness of $0.5 \mathrm{~mm}$ producing an effective energy of approximately $83 \mathrm{keV}$. The dishes holding the embryos were placed at a distance of $580 \mathrm{~mm}$ from the X-ray target, which received X-ray doses at a dose rate of about $1 \mathrm{~Gy} / \mathrm{min}$.

(A) The objective of the first part was to explore the conditions for successful induction of the bystander effect in naive embryos by partnering them with the irradiated embryos in the same medium. Dechorionated zebrafish embryos at $5 \mathrm{hpf}$ were divided into 5 groups, namely,

(1) Control group of embryos ( sample size $=10$ ), which received no further treatment after dechorionation;

(2) Irradiated group of embryos (sample size $=50$ ), which were irradiated with 4 Gy of X-ray at $5 \mathrm{hpf}$, and which were partnered with the bystander group of embryos immediately after irradiation;

(3) Sham-irradiated group of embryos (sample size $=50$ ), which were sham irradiated with 4 Gy of X-ray at $5 \mathrm{hpf}$, and which were partnered with the bystander control group of embryos immediately after sham irradiation;

(4) Bystander group of embryos (sample size $=10$ ), which were introduced into the dish hosting the irradiated group of embryos to partner with them immediately after irradiation until $25 \mathrm{hpf}$; and

(5) Bystander control group of embryos (sample size = 10), which were introduced into the dish hosting the sham-irradiated group of embryos to partner with them immediately after sham irradiation until 25 hpf.

The partnership of different groups of zebrafish embryos was schematically shown in Figure 1 . The two groups of embryos were accommodated into the two shallow dredged regions on the same agarose dish with $2.5 \mathrm{~mL}$ E3 medium $(5 \mathrm{mM} \mathrm{NaCl}$, $0.17 \mathrm{mM} \mathrm{KCl}, 0.33 \mathrm{mM} \mathrm{CaCl}, 0.33 \mathrm{mM} \mathrm{MgSO}$, $0.1 \%$ methylene blue). The soluble factors, if any, responsible for the communication of the bystander signal were expected to be released from the irradiated group of embryos to the bystander group of embryos. The mean number of apoptotic signals obtained for the bystander group of embryos, the bystander control group of embryos, and the control 


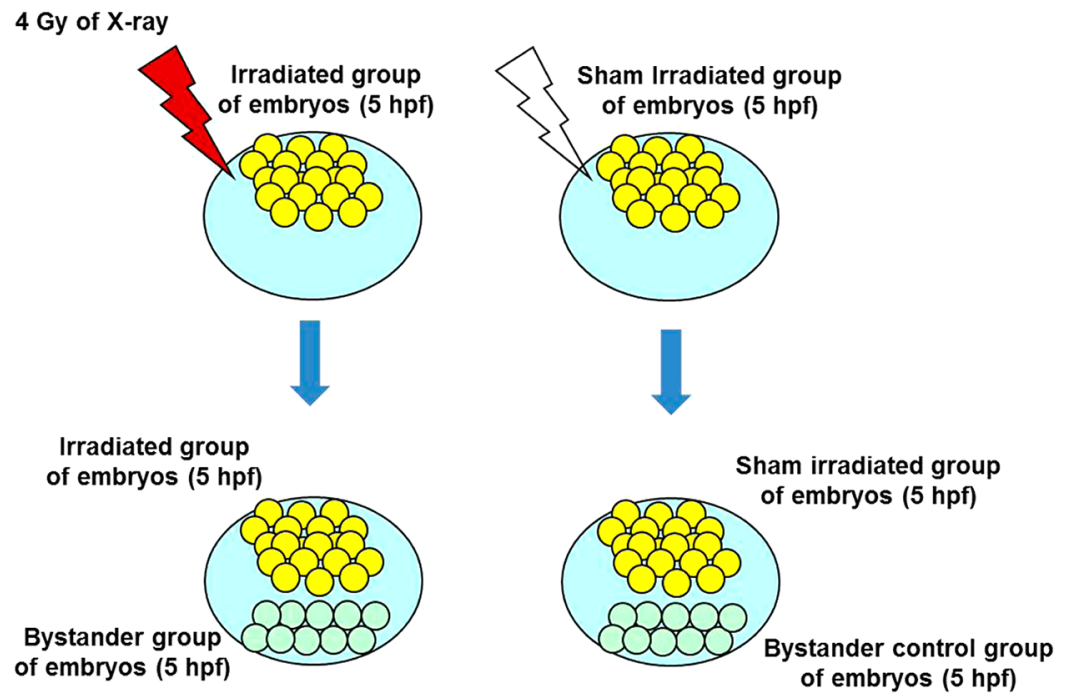

Figure 1. Schematic diagram showing the partnering of different groups of embryos in the same medium.

group of embryos at $25 \mathrm{hpf}$ were revealed through terminal dUTP transferase-mediated nick end-labeling (TUNEL) assay and captured using a confocal laser microscope as described by Choi et al. ${ }^{27}$ and also briefly described in the next session.

(B) The objective of the second part was to study the effects of adding the CO liberator CORM-3 with a concentration of $20 \mu \mathrm{M}$ or the NO scavenger cPTIO (Dojindo Molecular Technologies, INC., Kumamoto, Japan) with a concentration of $500 \mu \mathrm{M}^{28}$ into the medium on the induction of the bystander effect in naive embryos by partnering them with the irradiated embryos. CORM-3 was synthesized from commercially available tricarbonyldichlororuthenium(II) dimer (CORM-2 dimer, Sigma Aldrich) as described by Clark et al. ${ }^{29}$ To determine that the CORM-3 was active, we prepared inactive CORM-3 (iCORM3) as the negative control. ${ }^{30}$ The CORM-3 employed in the present work came from the same stock for our previous studies. ${ }^{30}$ All of the exposure steps were the same as part (A) except that either CORM-3 or cPTIO was added in the medium when the embryos were irradiated and until $25 \mathrm{hpf}$.

(C) The objective of the third part was to explore the conditions for successful induction of the bystander effect in naive embryos by introducing them into the irradiated embryo conditioned medium (IECM) alone, that is, without partnering with the irradiated embryos. The first batch of dechorionated zebrafish embryos at $5 \mathrm{hpf}$ were divided into two groups in two separate Petri dishes containing $2.5 \mathrm{~mL}$ of E3 medium, namely,

(1) Irradiated group of embryos (sample size $=50$ ), which were irradiated with $4 \mathrm{~Gy}$ of X-ray, then immediately placed into the incubator until $29 \mathrm{hpf}$, and of which the conditioned medium (i.e., the irradiated embryo conditioned medium or IECM) was harvested at $29 \mathrm{hpf}$;

(2) Sham-irradiated group of embryos (sample size = 50 ), which were sham irradiated with 4 Gy of $\mathrm{X}$-ray, then immediately placed into the incubator until $29 \mathrm{hpf}$, and of which the conditioned medium (i.e., the control embryo conditioned medium or CECM) was harvested at $29 \mathrm{hpf}$;
The second batch of dechorionated zebrafish embryos were collected at a time corresponding to $24 \mathrm{hpf}$ for the first batch of zebrafish embryos and were similarly dechorionated at $4 \mathrm{hpf}$. When the second batch of dechorionated zebrafish embryos developed into $5 \mathrm{hpf}$ stage, at which time the first batch of zebrafish embryos had developed into 29 hpf stage, the IECM or CECM were harvested. At the same time, the second batch of zebrafish embryos were divided into three groups in three separate Petri dishes, namely,

(3) Control group of embryos ( sample size $=10$ ), which received no further treatment after dechorionation;

(4) Bystander group of embryos (sample size $=10$ ), which were introduced into the dish containing IECM and returned into the incubator until $25 \mathrm{hpf}$; and

(5) Bystander control group of embryos (sample size = $10)$, which were introduced into the dish containing $\mathrm{CECM}$ and returned into the incubator until $25 \mathrm{hpf}$.

The schematic diagram showing the experimental schedule was shown in Figure 2. The mean number of apoptotic signals obtained for the bystander group of embryos, the bystander control group of embryos, and the control group of embryos at $25 \mathrm{hpf}$ were revealed through TUNEL assay and captured using a confocal microscope as before.

(D) The objective of the fourth part was to examine the effect of dilution of the IECM prepared as described for part (C) above on the induction of the bystander effect in naive embryos. All of the steps were the same as part (C) except that the bystander groups of embryos (sample size $=$ 10 ) and the bystander control groups of embryos (sample size $=10$ ) were introduced into dishes containing IECM and CECM respectively with different dilution factors and returned into the incubator until 25 hpf. Dilution factors of $2 \times$ and $5 \times$ were used to generate various concentrations of IECM or CECM (50\% and $20 \%)$. The schematic diagram showing the experimental schedule was shown in Figure 3.

TUNEL Assay. The response of naive zebrafish embryos to the partnered irradiated embryos or to the IECM was assessed 


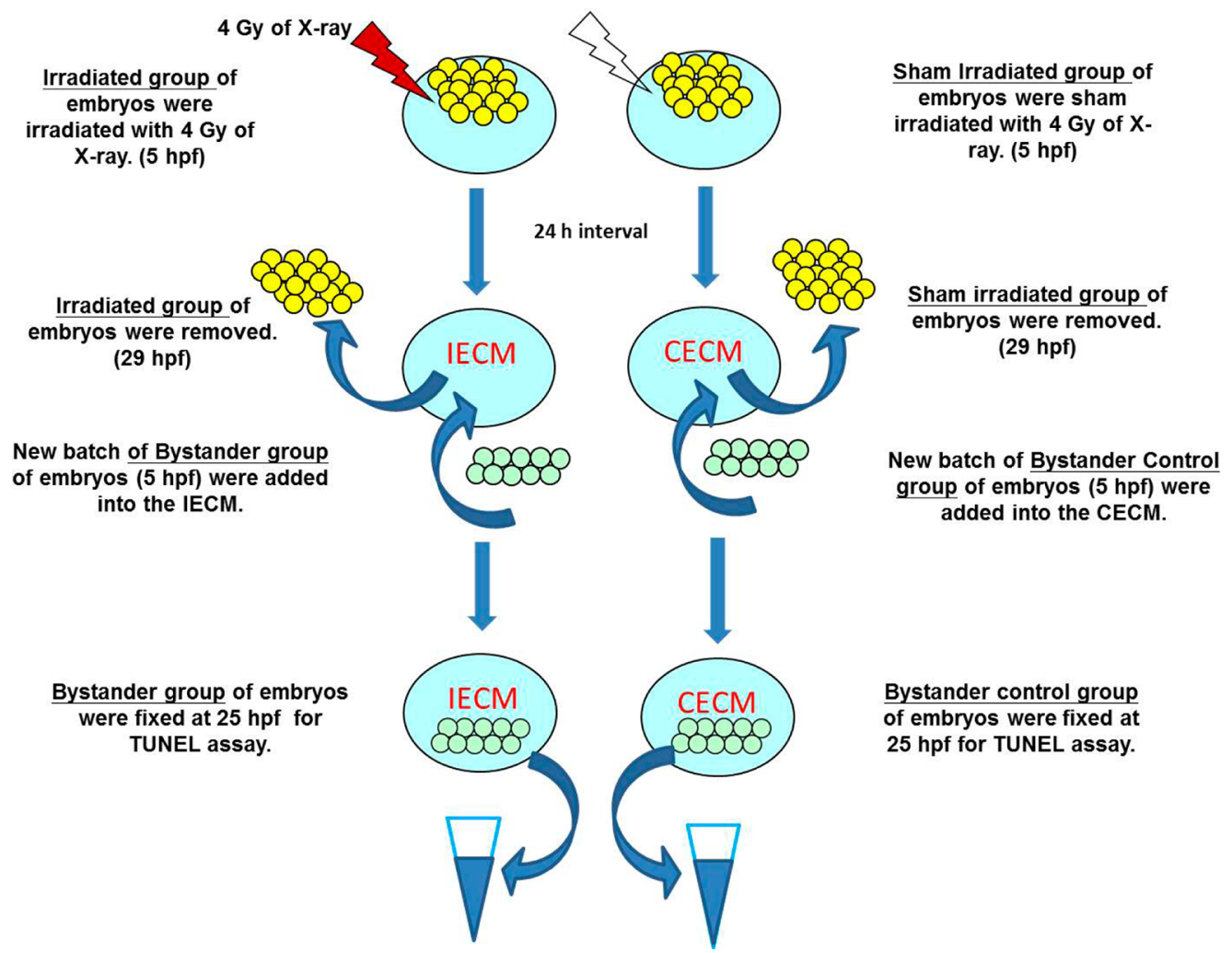

Figure 2. Schematic diagram showing the experimental schedule for part $\mathrm{C}$ of the experiment, which was designed to study the medium-mediated bystander effect through the introduction of the bystander group and bystander control group of embryos into the IECM or CECM, respectively.

through the number of apoptotic signals. Quantification of apoptotic signals has been commonly employed to analyze the radiation effect on the whole embryos. ${ }^{17,21,31,32}$ In the present work, apoptotic cells in naive embryos were revealed using the terminal dUTP transferase-mediated nick end-labeling (TUNEL) assay. ${ }^{27,33}$ The embryos were fixed in $4 \%$ paraformaldehyde in phosphate buffered saline (PBS) with $0.1 \%$ Tween 20 at room temperature for $5 \mathrm{~h}$. The fixed embryos were then dehydrated, and were then rehydrated and treated with $20 \mu \mathrm{g} / \mathrm{mL}$ protease kinase (Wako Pure Chemical Industries Ltd., Osaka, Japan) for $10 \mathrm{~min}$. The embryos were then fixed in $4 \%$ paraformaldehyde in PBS with $0.1 \%$ Tween 20 again. The TUNEL staining was carried out using an in situ apoptosis detection kit (MK500, Takara Bio. Inc., Japan). The fixed embryos were immersed in the permeabilization buffer for $30 \mathrm{~min}$ on ice. The apoptotic cells were labeled by staining the embryos in the mixture of Terminal Deoxynucleotidyl Transferase $(\mathrm{TdT})$ enzyme and labeling safe buffer containing fluorescein-labeled 2'-deoxyuridine, 5'-triphosphate, FITCdUTP in the ratio of 1 to 9 . The embryos were then incubated in a $37{ }^{\circ} \mathrm{C}$ humidified chamber for $110 \mathrm{~min}$ and then finally washed thoroughly by PBS in $0.1 \%$ Tween 20 . The apoptotic signals were captured by a confocal laser microscope (FV-1000, Olympus Corporation, Tokyo) with $4 \times$ objective lens (NA:0.16, UPLSAPO $4 \times$, Olympus Corporation, Tokyo). For each embryo, a total of 15 to 20 sliced images $(2.12 \times 2.12 \mathrm{~mm}$, $2.06 \mu \mathrm{m} /$ pixel) were captured with $25 \mu \mathrm{m}$ intervals from top to bottom of the embryo. The feasibility of apoptosis detection by TUNEL assay was described in our previous work. ${ }^{27}$

We did not perform TUNEL assay on the 4 Gy irradiated embryos because we were only interested in the bystander effect induced in unirradiated zebrafish embryos in the current study. The effect of ionizing radiation on (irradiated) zebrafish embryos has already been extensively studied in previous researches. For examples, Bladen et al. ${ }^{17}$ studied the DNA damage response and Ku80 mRNA function in the zebrafish embryos irradiated with ${ }^{137} \mathrm{Cs} \gamma$ rays. McAleer et al. ${ }^{18}$ evaluated the effects of $250 \mathrm{kVp} \mathrm{X}$-rays in combination with a known radioprotector (free radical scavenger Amifostine) or radiosensitizing agent (tyrosine kinase inhibitor AG1478) with a view to validate zebrafish embryos as a screen for radiation modifiers. McAleer et al. ${ }^{19}$ also used zebrafish embryos to study radiosensitizing effects of flavopiridol in normal tissues exposed to ${ }^{137} \mathrm{Cs} \gamma$ rays or $250 \mathrm{kVp}$ X-rays. Daroczi et al. ${ }^{20}$ evaluated the radioprotective effect of the nanoparticle DF-1, which was a fullerene with antioxidant properties in zebrafish embryos exposed to ${ }^{137} \mathrm{Cs} \gamma$ rays. Moreover, at high doses, the apoptotic signals seen as fluorescent dots in the images obtained by confocal laser microscope actually overlapped with one another due to the high density, which made the scoring difficult. ${ }^{27}$ As such, a study on the effect of ionizing radiation on irradiated zebrafish embryos was outside the scope of the present study.

Data Analysis. The number of apoptotic signals on each embryo after TUNEL assay was counted using the Image software freely obtainable from the Web site http://rsb.info.nih. gov/ij/. All experiments were repeated three times with a minimum of three replicates per experiment. To combine the data from different experiments, we first transformed the number of apoptotic signals for the bystander group of embryos $\left(N_{\mathrm{B}}\right)$ and for the bystander control group of embryos $\left(N_{\mathrm{BC}}\right)$ to the normalized net number of apoptotic signals (NAS) for the bystander group of embryos $\left(N_{\mathrm{B}} *\right)$ and for the bystander control group of embryos $\left(N_{\mathrm{BC}} *\right)$ as $N_{\mathrm{B}} *=\left[N_{\mathrm{B}}-\left\langle N_{\mathrm{CTRL}}\right\rangle\right] /\left\langle N_{\mathrm{CTRL}}\right\rangle$ and $N_{\mathrm{BC}} *=\left[N_{\mathrm{BC}}-\left\langle N_{\mathrm{CTRL}}\right\rangle\right] /\left\langle N_{\mathrm{CTRL}}\right\rangle$ respectively where 

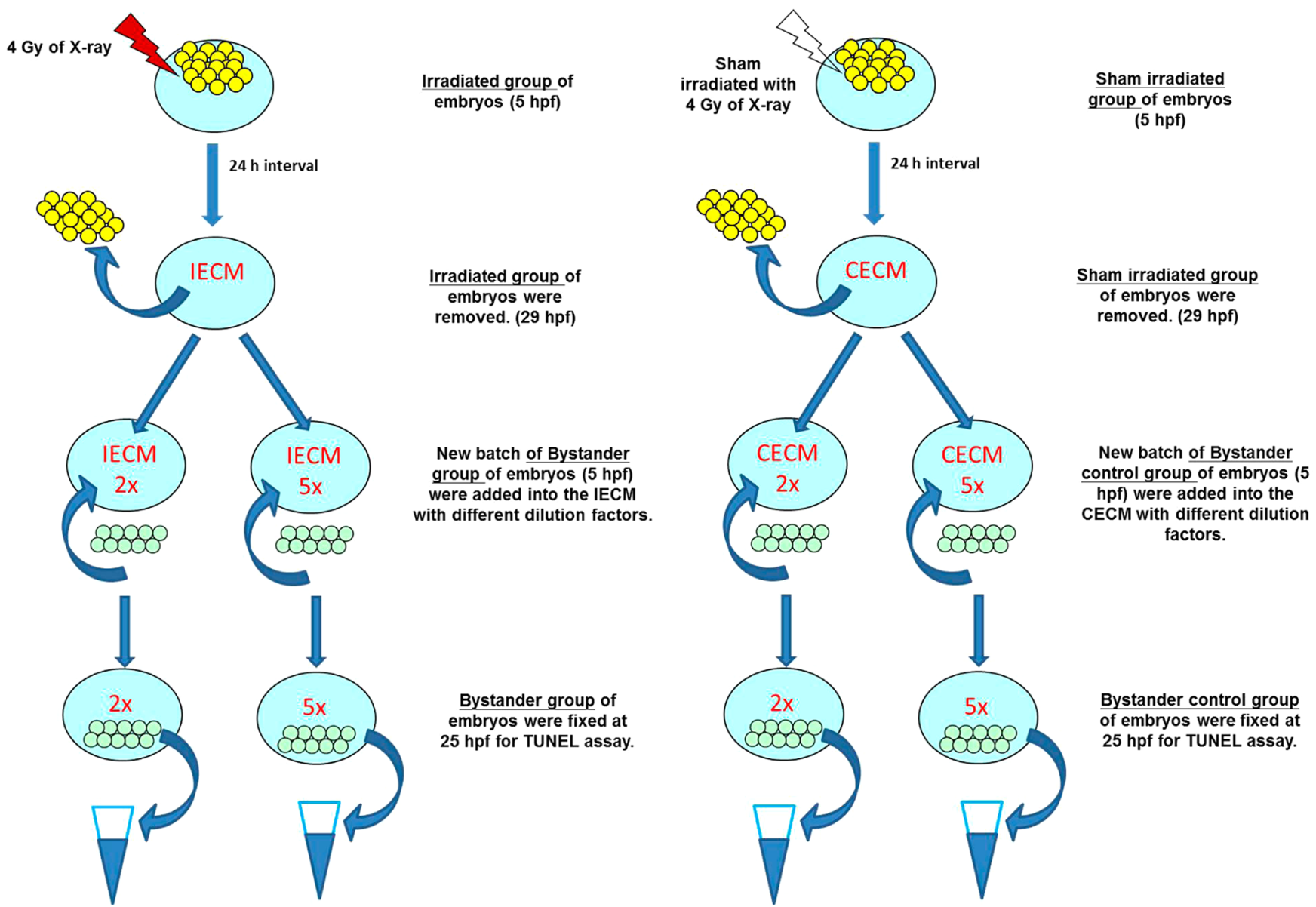

Figure 3. Schematic diagram showing the experimental schedule for part $\mathrm{D}$ of the experiment, which was designed to study the dilution effect of the IECM and CECM on the induction of the bystander effect.

$\left\langle N_{\text {CTRL }}\right\rangle$ was the average apoptotic signals for the corresponding control group of embryos. The statistical significance for differences between the compared groups was obtained through $t$ tests, and $p$ values $<0.05$ were considered correspond to statistically significant differences. The relative NAS was defined as $\left(N_{\mathrm{B}} *-N_{\mathrm{BC}} *\right)$.

\section{RESULTS}

The results for the four parts of our experiments are as follows.

(A) We explored the conditions for successful induction of the bystander effect in naive embryos by partnering them with the irradiated embryos. There were a total of 5 separate sets of experimental data. We have provided as an example the raw data for the number of apoptotic signals before normalization as part A of the Supporting Information. The endogenous apoptosis signal on a $25 \mathrm{hpf}$ zebrafish embryos was 27, which was obtained from the average of the apoptotic signals from the five different set of control embryos. The relative NAS value is shown in Figure 4. The data showed that the apoptotic signals of the bystander group of embryos were significantly more than those of the bystander control group of embryos with a $p$ value of $8.6 \times$ $10^{-4}$. As such, significant bystander response was induced when 10 naive embryos were partnered with 50 irradiated embryos (irradiated with $4 \mathrm{~Gy}$ of X-rays at $5 \mathrm{hpf}$ ) immediately after irradiation until $25 \mathrm{hpf}$.

(B) We studied the effects of adding the $\mathrm{CO}$ liberator CORM-3 or the NO scavenger cPTIO into the medium

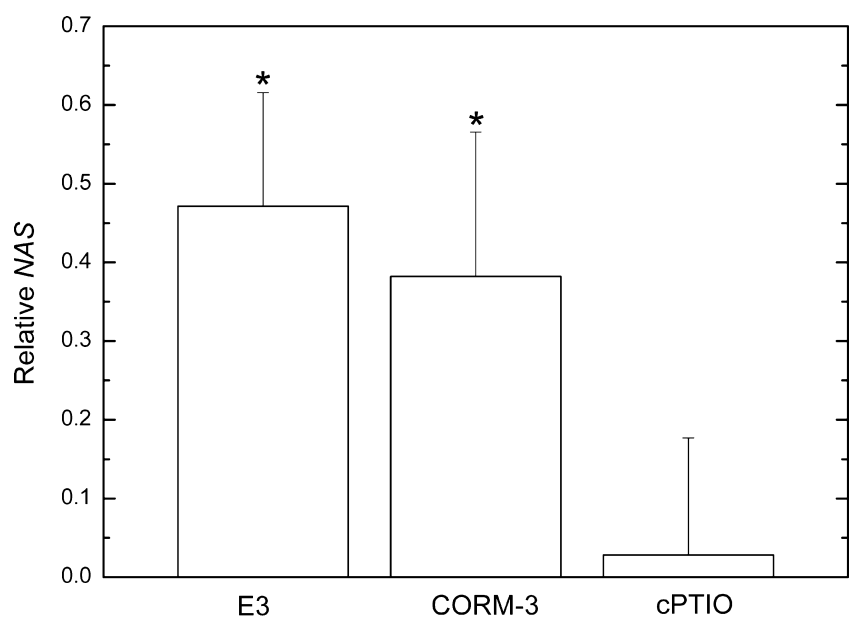

Figure 4. Relative NAS values for naive embryos which had partnered with irradiated embryos in E3 solution (E3), in E3 solution with the CO liberator CORM-3 (CORM-3) and in E3 solution with the NO scavenger cPTIO (cPTIO). The differences of these samples from the corresponding bystander controls were examined using the $t$ test. An asterisk represents a statistically significant difference where $p<0.05$.

on the induction of the bystander effect in naive embryos by partnering them with the irradiated embryos. There were a total of 3 separate sets of experimental data for each of CORM-3 and cPTIO. The relative NAS values are shown in Figure 4. The results showed that the 
apoptotic signals of the bystander group of embryos treated with CORM-3 were significantly larger than that of the bystander control group of embryos with a $p$ value of 0.023 . In other words, CORM-3 failed to suppress the bystander response induced through $\mathrm{X}$-ray irradiation. However, no significant difference was found between the bystander group and the bystander control group under cPTIO treatment $(p=0.43)$. In other words, cPTIO successfully suppressed the bystander response induced through X-ray.

(C) With a view to elucidate the involvement of long-lasting bystander signals in the medium-mediated bystander effect, we explored the conditions for successful induction of the bystander effect in naive embryos by introducing them into the IECM alone. There were a total of 4 separate sets of experimental data. The relative NAS value is shown in Figure 5. The data showed that

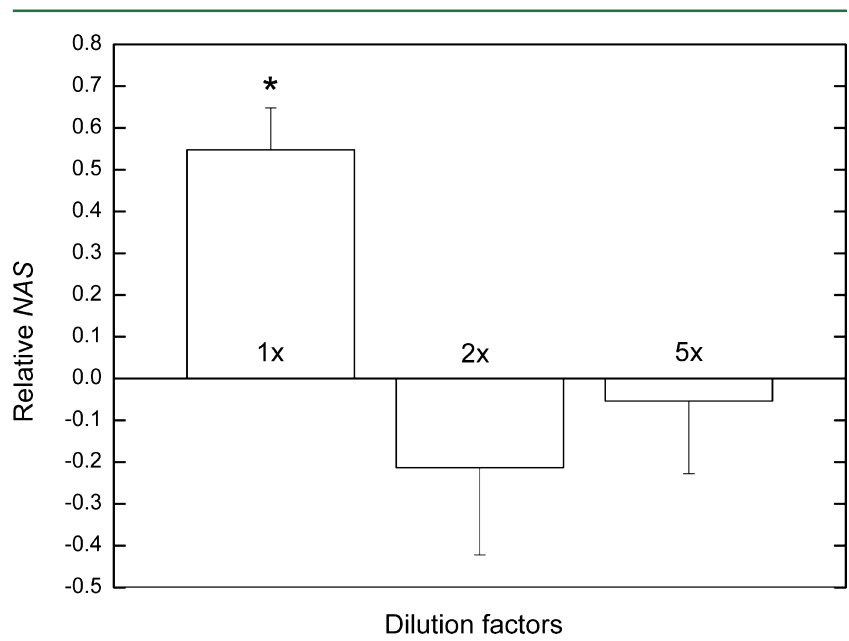

Figure 5. Relative NAS values for naive embryos which had been introduced into the irradiated embryo conditioned medium (IECM) alone without partnering with the irradiated embryos, with different dilution factors of the IECM. The differences of these samples from the corresponding bystander controls were examined using the $t$ test. An asterisk represents a statistically significant difference where $p<0.05$.

the apoptotic signal of the bystander group of embryos were significantly larger than that of the bystander control group of embryos with a $p$ value of $1.1 \times 10^{-6}$. As such, significant bystander response was induced when 10 naive embryos were introduced into the IECM alone, without the presence of the irradiated embryos, and returned into the incubator until $25 \mathrm{hpf}$. This showed that bystander effect could still be induced even if the 50 irradiated embryos (irradiated with $4 \mathrm{~Gy}$ of X-rays) were not partnered with the naive embryos and suggested that some long-lasting bystander signals were present in the medium. The data point here corresponded to a dilution factor of $1 \times$.

(D) We examined the effect of dilution of the IECM prepared as described for part (C) above on the induction of the bystander effect in naive embryos. There were a total of 4 separate sets of experimental data for each dilution factor of $2 \times$ and $5 \times$. The relative NAS values for dilution factors of $2 \times$ and $5 \times$ together with that for the dilution factor of $1 \times$ (from part (C) above) are shown in Figure 5. The results showed that the bystander group of embryos introduced into the IECM having been diluted by $2 \times$ and $5 \times$ did not show significant differences in apoptotic signals when compared with the bystander control group $(p=0.16$ and 0.38$)$. These showed that no significant bystander responses were induced for these dilution factors.

\section{DISCUSSION}

There has been only a scarce amount of studies on radiation induced bystander effect (RIBE) on the interorganism level including the study on RIBE between mice by Surinov et al., ${ }^{2}$ and study on RIBE between whole fish by Mothersill's group ${ }^{4,5}$ as described in the Introduction.

The first part of the present study demonstrated the successful induction of the bystander effect in 10 naive embryos by partnering them with the 50 irradiated embryos (irradiated with $4 \mathrm{~Gy}$ of X-rays at $5 \mathrm{hpf}$ ) in the same medium immediately after irradiation until $25 \mathrm{hpf}$. This was the very first observation of high-dose bystander effect between organisms. RIBE has been extensively studied at low doses because evidence of the bystander-induced cell killing and other effects on unirradiated cells were found to be predominant at doses up to $0.5 \mathrm{~Gy}$. High-dose bystander effect was only reported very recently and only in cell lines. ${ }^{15,16}$ The apoptotic signal in the bystander group was found to have increased by $47 \%$ when compared with the bystander control group. The results showed that the bystander effect was mediated by a factor(s) that could be transferred in medium and agreed with our previous studies where zebrafish embryos irradiated with alpha particles were able to communicate the induced stress to partnered naive embryos sharing the same medium to increase cell deaths, ${ }^{34}$ to induce $\mathrm{RAR}^{6}$ or to induce hormetic effect ${ }^{10}$ in the naive embryos. Choi et al. ${ }^{35}$ further demonstrated the rescue effect where the stress communicated between the unirradiated zebrafish embryos and the irradiated embryos sharing the same medium successfully helped the irradiated embryos mitigate the radiation induced DNA damage. Choi et al. ${ }^{6,10}$ argued that the bystander effect induced in the naive embryos leading to lowdose radiation effects such as RAR and hormetic effect supported the view that radiation-induced stress communicated in vivo between living organisms were actually an allelopathic effect aimed at coordinating a species-level survival response ${ }^{3}$ at least in aquatic species that were close to one another and sharing the same media.

The second part of the present study revealed that the NO scavenger cPTIO succeeded, whereas the CO liberator CORM3 failed to suppress the bystander response induced through $\mathrm{X}$ ray irradiation. These results shed light on the nature of chemical messengers responsible for inducing the bystander effect in naive embryos. Our result from cPTIO showed that $\mathrm{NO}$ was involved in the bystander response between zebrafish embryos induced through X-ray irradiation. NO is a component of many signaling pathways, and induction of inducible nitric oxide synthase (iNOS) was identified as an early signaling event in response to ionizing radiation. ${ }^{36} \mathrm{NO}$ was also considered a chemical factor in inducing the bystander effect. ${ }^{37,38}$ Intercellular induction of apoptosis through $\mathrm{NO} /$ peroxynitrite signaling pathway from the interaction of $\mathrm{NO}$ with superoxide anions was also previously reported by Bauer. ${ }^{39}$ A series of studies carried out by Shao et al. ${ }^{40-43}$ indicated that NO was a component of the signaling pathway leading to bystander effects. Anzenberg et al. ${ }^{44}$ found that the NO scavenger PTIO 
was effective in blocking all bystander effects in the AG01522 fibroblasts.

However, our results showed that $\mathrm{CO}$ did not suppress the RIBE induced between zebrafish embryos. For a comparison, Choi et al. ${ }^{30}$ demonstrated that a low concentration of exogenous CO liberated from CORM-3 (from 5 to $20 \mu \mathrm{M}$ ) suppressed $\alpha$-particle-induced bystander effect between zebrafish embryos in vivo in a CO-concentration dependent manner, but CORM-3 with a concentration of $5 \mu \mathrm{M}$ was not strong enough to entirely suppress the RIBE. Han et al. ${ }^{45}$ also discovered the mitigation of in vitro RIBE by low-concentration $\mathrm{CO}$ in a concentration-dependent manner. Subsequently, Han et al. ${ }^{46}$ found evidence showing that the mitigation was initiated by impairing the response of the bystander cells to NO rather than by affecting the release of NO by the irradiated cells. As such, although we previously studied the role of $\mathrm{CO}$ on the effects of alpha particles on zebrafish embryos, the results were different from the present study using X-rays. The different conclusions between the present work involving X-rays and the previous work involving $\alpha$ particles might be attributed to the high and low linear energy transfers (LETs) of $\alpha$ particles and $\mathrm{X}$-rays, respectively. It was established that $\alpha$-particle irradiation led to formation of a larger amount of clustered DNA lesions when compared to X-ray irradiation, which were potentially more difficult to repair and more susceptible to mutagenic changes. ${ }^{47}$ Pinto et al. ${ }^{48}$ reported that the majority of the DNA double-strand breaks (DSBs) in primary human fibroblasts caused by X-ray irradiation were removed after $24 \mathrm{~h}$, whereas $\sim 85 \%$ of those caused by $\alpha$-particle irradiation remained. Thomas et al. ${ }^{49}$ revealed that $\alpha$-particle irradiation was 7 to 14 times more effective than X-rays in killing bovine aortic endothelial cells. In particular, Chauhan et al. ${ }^{50}$ discovered that expression of Fas and TNF- $\alpha$ occurred in human monocytic THP-1 cells after being irradiated with $\alpha$ particles and X-rays with doses from 0 to $1.5 \mathrm{~Gy}$ but with expression levels of TNF- $\alpha$ significantly higher for $\alpha$-particle irradiation. TNF- $\alpha$ could activate the NF- $\kappa \mathrm{B} / \mathrm{COX}-2$ signal pathway to induce inflammation-type effect in bystander cells while cyclooxygense-2 (COX-2) was identified as a central component of the bystander signaling scheme. ${ }^{51} \mathrm{NF}-\kappa \mathrm{B}$ enters the nucleus and acts as a transcription factor for COX-2 and iNOS genes. CO might suppress RIBE through inhibition of the COX-2 signaling pathway ${ }^{52}$ and attenuation of activation of NF- $\kappa B^{53}$ However, the concentration $(20 \mu \mathrm{M})$ of $\mathrm{CO}$ used in this study was much less than the concentration, which could effectively manipulate the NOS system. ${ }^{54}$ Han et al. ${ }^{46}$ also showed that the expression or activity of NOS in the irradiated cells were not affected by a similar concentration $(14 \mu \mathrm{M})$ of $\mathrm{CO}$ through direct measurement of generated NO with the fluorescence probe. Anzenberg et al. ${ }^{44}$ demonstrated the LET-dependent differences in the signal released from DU-145 human prostate carcinoma cells by irradiating them with either $\alpha$ particles or $250 \mathrm{kVp}$ X-rays. They showed that the X-irradiated tumor cells succeeded while alpha-particle-irradiated tumor cells failed to cause medium-mediated bystander effects in cocultured unirradiated AG01522 human fibroblasts (in terms of decrease in the surviving fraction and increase in $\gamma-\mathrm{H} 2 \mathrm{AX}$ focus induction). However, it is also remarked here that different irradiated cells could also release different signals into the medium. Yang et al. ${ }^{55,56}$ used the same bystander cells and end points as those of Anzenberg et al. ${ }^{44}$ but used a different high-LET radiation $(1 \mathrm{GeV} /$ nucleon iron ions with LET of $151 \mathrm{keV} / \mu \mathrm{m}$ ) and different directly irradiated cells (AG01522 human fibroblasts). These authors concluded that LET did not affect the bystander effect in terms of $\gamma$-H2AX formation, $\mathrm{MN}$ induction, or the surviving fraction of the bystander cells. Future studies on whether and how ionizing radiations with LETs lead to different responses in irradiated and bystander zebrafish embryos would be interesting and pertinent.

The third part of the present study showed the successful induction of the bystander effect in naive embryos by introducing them into the irradiated embryo conditioned medium (IECM) alone, without the presence of the irradiated embryos, and returned into the incubator until $25 \mathrm{hpf}$. The apoptotic signal in the bystander embryos increased by $55 \%$. This suggested that some long-lasting bystander signals were present in the medium and involved in the induction of the bystander effect. NO from the irradiated embryos was unlikely to be involved here because of its short life. Nevertheless, downstream products of signaling cascades induced by NO might still be involved.

The fourth part of the present study illustrated that no significant bystander responses were induced in naive embryos introduced into the IECM having been diluted by $2 \times$ and $5 \times$. Moreover, although not significant, it was interesting that both the results for $2 \times$ and $5 \times$ dilutions of the ICEM appeared to trend to an overall reduction in apoptosis, which looked rather like an adaptive response. These results reminded us about the observation of Ryan et al. ${ }^{57}$ from their in vitro experiments regarding the effect of dilution of the irradiated cell conditioned medium (ICCM). According to the argument put forward by Ryan et al., 57 the rapid abolishment of the bystander effect by the dilution of the factor(s) in the ICCM suggested that the bystander effect was likely an on/off response with a threshold in activating the bystander response below which no response is triggered. It is remarked that our present work was the first in vivo study showing results that agreed with the proposal that the bystander effect was an on/off response with a threshold. Such a phenomenon had only been observed in vitro studies. ${ }^{57}$ The proposal of a dose threshold for triggering a bystander effect agreed with a previous study on $\gamma$-ray-induced bystander effect. $^{58}$

\section{ASSOCIATED CONTENT}

\section{Supporting Information}

Experimental results showing the number of apoptotic signals obtained from the three groups of zebrafish embryos in the five separate experiments of Part A have been provided as an example for the number of apoptotic signals before normalization. This material is available free of charge via the Internet at http://pubs.acs.org.

\section{AUTHOR INFORMATION}

\section{Corresponding Author}

*E-mail: peter.yu@cityu.edu.hk, tel: +852-34427812, fax: +85234420538 .

\section{Notes}

The authors declare no competing financial interest.

\section{ACKNOWLEDGMENTS}

The adult zebrafish employed in the present research were kindly provided by RIKEN Brain Science Institute, JAPAN (courtesy Prof. Hitoshi Okamoto). Animal ethics procedures have been approved. Operation and dose measurements of $\mathrm{X}$-ray generator were supported by the staff of the Radiation Engineering and Technical Support section of NIRS. V.W.Y. 
Choi and C.Y.P. $\mathrm{Ng}$ would like to acknowledge the great hospitality provided by the staff of NIRS during their stay.

\section{REFERENCES}

(1) Garnier-Laplace, J.; Beaugelin-Seiller, K.; Hinton, T. G. Fukushima wildlife dose reconstruction signals ecological consequences. Environ. Sci. Technol. 2011, 45, 5077-5078.

(2) Surinov, B. P.; Isaeva, V. G.; Dukhova, N. N. Post radiation immunosuppressive and attractive volatile secretions: the "bystander effect" or allelopathy in groups of animals. Dokl. Biol. Sci. 2005, 400, 28-30.

(3) Mothersill, C.; Bucking, C.; Smith, R. W.; Agnihotri, N.; O’Neill, A.; Kilemade, M.; Seymour, C. B. Communication of radiation induced stress or bystander signals between fish in vivo. Environ. Sci. Technol. 2006, 40, 6859-64.

(4) Mothersill, C.; Smith, R. W.; Agnihotri, N.; Seymour, C. B. Characterization of a radiation-induced stress response communicated in vivo between zebrafish. Environ. Sci. Technol. 2007, 41, 3382-3387.

(5) Mothersill, C.; Smith, R. W.; Hinton, T. G.; Aizawa, K.; Seymour, C. B. Communication of radiation-induced signals in vivo between DNA repair deficient and proficient medaka (Oryzias latipes). Environ. Sci. Technol. 2009, 43, 3335-3342.

(6) Choi, V. W. Y.; Cheng, S. H.; Yu, K. N. Radioadaptive response induced by alpha-particle-induced stress communicated in vivo between zebrafish embryos. Environ. Sci. Technol. 2010, 44, 88298834 .

(7) Olivieri, G.; Bodycote, Y.; Wolff, S. Adaptive response of human lymphocytes to low concentrations of radioactive thymidine. Science 1984, 223, 594-597.

(8) Cai, L.; Jiang, J.; Wang, B.; Yao, H.; Wang, X. Induction of an adaptive response to dominant lethality and to chromosome damage of mouse germ cells by low dose radiation. Mutat. Res. 2003, 303, $157-161$.

(9) Wang, B.; Ohyama, H.; Shang, Y.; Tanaka, K.; Aizawa, S.; Yukawa, O.; Hayata, I. Adaptive response in embryogenesis: V. Existence of two efficient dose-rate ranges for 0.3 Gy of priming irradiation to adapt mouse fetuses. Radiat. Res. 2004, 161, 264-272.

(10) Choi, V. W. Y.; Cheung, A. L. Y.; Cheng, S. H.; Yu, K. N. Hormetic effect induced by alpha-particle-induced stress communicated in vivo between zebrafish embryos. Environ. Sci. Technol. 2012, 46, 11678-11683.

(11) Calabrese, E. J.; Baldwin, L. A. Defining hormesis. Hum. Exp. Toxicol. 2002, 21, 91-97.

(12) Calabrese, E. J.; Linda, A. B. Toxicology rethinks its central belief. Nature 2003, 421, 691-692.

(13) Calabrese, E. J. Hormesis: Why it is important to toxicology and toxicologists? Environ. Toxicol. Chem. 2008, 27, 1451-1474.

(14) Kadhim, M. A.; Moore, S. R; Goodwin, E. H. Interrelationships amongst radiation-induced genomic instability, bystander effects, and the adaptive response. Mutat. Res. 2004, 568, 21-32.

(15) Shareef, M .M.; Cui, N.; Burikhanov, R.; Cupta, S.; Satishkumar, S.; Shajahan, S.; Mohiuddin, M.; Rangnekar, V. M.; Ahmed, M. M. Role of tumor necrosis factor- $\alpha$ and TRAIL in high-dose radiationinduced bystander signaling in lung adenocarcinoma. Cancer Res. 2007, 67, 11811-11820.

(16) Asur, R. S.; Sharma, S.; Chang, C. W.; Penagaricano, J.; Kommuru, I. M.; Moros, E. G.; Corrya, P. M.; Griffina, R. J. Spatially fractionated radiation induces cytotoxicity and changes in gene expression in bystander and radiation adjacent murine carcinoma cells. Radiat. Res. 2012, 177, 751-765.

(17) Bladen, C. L.; Lam, W. K.; Dynan, W. S.; Kozlowski, D. J. DNA damage response and Ku80 function in the vertebrate embryo. Nucleic Acids Res. 2005, 33, 3002-3010.

(18) McAleer, M. F.; Davidson, C.; Davidson, W. R.; Yentzer, B.; Farber, S. A.; Rodeck, U.; Dicker, A. P. Novel use of zebrafish as a vertebrate model to screen radiation protectors and sensitizers. Int. J. Radiat. Oncol., Biol., Phys. 2005, 61, 10-13.

(19) McAleer, M. F.; Duffy, K. T.; Davidson, W. R.; Kari, G.; Dicker, A. P.; Rodeck, U.; Wickstrom, E. Antisense inhibition of cyclin D1
Expression is equivalent to flavopiridol for radiosensitization of zebrafish embryos. Int. J. Radiat. Oncol., Biol., Phys. 2006, 66, 546-551. (20) Daroczi, B.; Kari, G.; McAleer, M. F.; Wolf, J. C.; Rodeck, U.; Dicker, A. P. In vivo radioprotection by the fullerene nanoparticle DF1 as assessed in a zebrafish model. Clin. Cancer Res. 2006, 12, 70867091.

(21) Geiger, G. A.; Parker, S. E.; Beothy, A. P.; Tucker, J. A.; Mullins, M. C.; Kao, G. D. Zebrafish as a "Biosensor"? Effects of ionzing radiation and amifostine on embryonic viability and development. Cancer Res. 2006, 66, 8172-8181.

(22) Yum, E. H. W.; Choi, V. W. Y.; Nikezic, D.; Li, V. W. T.; Cheng, S. H.; Yu, K. N. Alpha-particle-induced bystander effects between zebrafish embryos in vivo. Radiat. Meas. 2009, 44, 1077-1080.

(23) Choi, V. W. Y.; Wong, M. Y. P.; Cheng, S. H.; Yu, K. N. Dosimetric study of radioadaptive response of zebrafish embryos using PADC-film substrates. Radiat. Meas. 2011, 46, 1795-1798.

(24) Yu, K. N.; Tung, M. M. T.; Choi, V. W. Y.; Cheng, S. H. Alpha radiation exposure decreases apoptotic cells in zebrafish embryos subsequently exposed to the chemical stressor, Cd. Environ. Sci. Pollut. Res. 2012, 19, 3831-3839.

(25) Barbazuk, W. B.; Korf, I.; Kadavi, C.; Heyen, J.; Tate, S.; Wun, E.; Bedell, J. A.; McPherson, J. D.; Johnson, S. L. The syntenic relationship of the zebrafish and human genomes. Genome Res. 2000, 10, 1351-1358.

(26) Choi, V. W. Y.; Lam, R. K. K.; Chong, E. Y. W.; Cheng, S. H.; $\mathrm{Yu}, \mathrm{K} . \mathrm{N}$. Designing experimental setup and procedures for studying alpha-particle-induced adaptive response in zebrafish embryos in vivo. Nucl. Instrum. Methods B 2010, 268, 651-656.

(27) Choi, V. W. Y.; Konishi, T.; Oikawa, M.; Iso, H.; Cheng, S. H.; $\mathrm{Yu}, \mathrm{K} . \mathrm{N}$. Adaptive response in zebrafish embryos induced using microbeam protons as priming dose and X-ray photons as challenging dose. J. Radiat. Res. 2010, 51, 657-664.

(28) Lepiller, S.; Laurens, V.; Bouchot, A.; Herbomel, P.; Solary, E.; Chluba, J. Imaging of nitric oxide in a living vertebrate using a diaminofluorescein probe. Free Radical Biol. Med. 2007, 43, 619-627.

(29) Clark, J. E.; Naughton, P.; Shurey, S.; Green, C. J.; Johnson, T. R.; Mann, B. E.; Foresti, R.; Motterlini, R. Cardioprotective actions by a water-soluble carbon monoxide-releasing molecule. Circ. Res. 2003, 93, e2-e8.

(30) Choi, V. W. Y.; Wong, M. Y. P.; Cheng, S. H.; Yu, K. N. Effects of exogenous carbon monoxide on radiation-induced bystander effect in zebrafish embryos in vivo. Appl. Radiat. Isot. 2012, 70, 1075-1079.

(31) Bladen, C. L.; Navarre, S.; Dynan, W. S.; Kozlowski, D. J. Expression of the Ku70 subunit (XRCC6) and protection from low dose ionizing radiation during zebrafish embryogenesis. Neurosci. Lett. 2007, 422, 97-102.

(32) Bladen, C. L.; Flowers, M. A.; Miyake, K.; Podolsky, R. H.; Barrett, J. T.; Kozlowski, D. J.; Dynan, W. S. Quantification of ionizing radiation-induced cell death in situ in a vertebrate embryo. Radiat. Res. 2007, 168, 149-157.

(33) Choi, V. W. Y.; Yum, E. H. W.; Konishi, T.; Oikawa, M.; Cheng, S. H.; Yu, K. N. Triphasic low-dose response in zebrafish embryos irradiated by microbeam protons. J. Radiat. Res. 2012, 53, 475-481.

(34) Yum, E. H. W.; Li, V. W. T.; Choi, V. W. Y.; Cheng, S. H.; Yu, K. N. Effects of alpha particles on zebrafish embryos. Appl. Radiat. Isot. 2010, 68, 714-717.

(35) Choi, V. W. Y.; Ng, C. Y. P.; Cheng, S. H.; Yu, K. N. $\alpha$-Particle irradiated zebrafish embryos rescued by bystander unirradiated zebrafish embryos. Environ. Sci. Technol. 2012, 46, 226-231.

(36) Leach, J. K.; Black, S. M.; Schmidt-Ullrich, R. K.; Mikkelsen, R. B. Activation of constitutive nitric-oxide synthase activity is an early signaling event induced by ionizing radiation. J. Biol. Chem. 2002, 277, 15400-15406.

(37) Azzam, E. I.; de Toledo, S. M.; Little, J. B. Stress signaling from irradiated to non-irradiated cells. Curr. Cancer Drug Targets 2004, 4, 53-64.

(38) Hamada, N.; Matsumoto, H.; Hara, T.; Kobayashi, Y. Intercellular and intracellular signaling pathways mediating ionizing 
radiation-induced bystander effects. J. Radiat. Res. (Tokyo) 2007, 48, 87-95.

(39) Bauer, G. Low dose radiation and intercellular induction of apoptosis: potential implications for the control of oncogenesis. Int. J. Radiat. Biol. 2007, 83, 873-888.

(40) Shao, C.; Furusawa, Y.; Aoki, M.; Matsumoto, H.; Ando, K. Nitric oxide-mediated bystander effect induced by heavy-ions in human salivary gland tumour cells. Int. J. Radiat. Biol. 2002, 78, 837844.

(41) Shao, C.; Stewart, V.; Folkard, M.; Michael, B. D.; Prise, K. M. Nitric oxide-mediated signaling in the bystander response of individually targeted glioma cells. Cancer Res. 2003, 63, 8437-8442.

(42) Shao, C.; Folkard, M.; Prise, K. M. Role of TGF- $\beta 1$ and nitric oxide in the bystander response of irradiated glioma cells. Oncogene 2007, 27, 434-440.

(43) Shao, C.; Prise, K. M.; Folkard, M. Signaling factors for irradiated glioma cells induced bystander responses in fibroblasts. Mutat. Res. 2008, 638, 139-145.

(44) Anzenberg, V.; Chandiramani, S.; Coderre, J. A. LET-dependent bystander effects caused by irradiation of human prostate carcinoma cells with $\mathrm{x}$ rays or alpha particles. Radiat. Res. 2008, 170, 467-476.

(45) Han, W.; Wu, L.; Chen, S.; Yu, K. N. Exogenous carbon monoxide protects the bystander chinese hamster ovary cells in mixed co-culture system after alpha-particle irradiation. Carcinogenesis 2010, $31,275-280$.

(46) Han, W.; Yu, K. N.; Wu, L. J.; Wu, Y. C.; Wang, H. Z. Mechanism of protection of bystander cells by exogenous carbon monoxide: Impaired response to damage signal of radiation-induced bystander effect. Mutat. Res. 2011, 709-710, 1-6.

(47) Hada, M.; Georgakilas, A. G. Formation of clustered DNA damage after high-LET irradiation: A review. J. Radiat. Res. 2008, 49, 203-210.

(48) Pinto, M.; Prise, K. M.; Michael, B. D. Double strand break rejoining after irradiation of human fibroblasts with X-rays or $\alpha$ particles: PFGE studies and numerical models. Radiat. Prot. Dosim. 2002, 99, 133-136.

(49) Thomas, P. A.; Tracy, B. L.; Ping, T.; Wickstrom, M.; Sidhu, N.; Hiebert, L. Relative biological effectiveness (RBE) of Po alphaparticles versus X-rays on lethality in bovine endothelial cells. Int. J. Radiat. Biol. 2003, 79, 107-118.

(50) Chauhan, V.; Howland, M.; Chen, J.; Kutzner, B.; Wilkins, R. C. Differential effects of alpha-particle radiation and $\mathrm{x}$-irradiation on genes associated with apoptosis. Radiology Research and Practice 2011, Article ID 679806, 9 pages.

(51) Hei, T. K.; Zhou, H.; Ivanov, V. N.; Hong, M.; Lieberman, H. B.; Brenner, D. J.; Amundson, S. A.; Gead, C. R. Mechanism of radiation-induced bystander effects: a unifying model. J. Pharm. Pharmacol. 2008, 60, 943-950.

(52) Alcaraz, M. J.; Fernandez, P.; Guillen, M. I. Anti-inflammatory actions of the heme oxygenase-1 pathway. Curr. Pharm. Des. 2003, 9, 2541-2551.

(53) Cepinskas, G.; Katada, K.; Bihari, A.; Potter, R. F. Carbon monoxide (CO) liberated from CO-releasing molecule (CORM-2) attenuates inflammation in the liver of septic mice. Am. J. Physiol. 2008, 294, G184-G191.

(54) Sarady, J. K.; Zuckerbraun, B. S.; Bilban, M.; Wagner, O.; Usheva, A.; Liu, F.; Ifedigbo, E.; Zamora, R.; Choi, A. M.; Otterbein, L. E. Carbon monoxide protection against endotoxic shock involves reciprocal effects on iNOS in the lung and liver. FASEB J. 2004, 18, 854-856.

(55) Yang, H.; Anzenberg, V.; Held, K. D. Effects of heavy ions and energetic protons on normal human fibroblasts. Radiat. Biol. Radioecol. 2007, 47, 302-306.

(56) Yang, H.; Anzenberg, V.; Held, K. D. The time dependence of bystander responses induced by iron-ion radiation in normal human skin fibroblasts. Radiat. Res. 2007, 168, 292-298.

(57) Ryan, L. A.; Smith, R. W.; Seymour, C. B.; Mothersill, C. E. Dilution of Irradiated Cell Conditioned Medium and the Bystander Effect. Radiat. Res. 2008, 169, 188-196.
(58) Liu, Z.; Mothersill, C. E.; McNeill, F. E.; Lyng, F. M.; Byun, S. H.; Seymour, C. B.; Prestwich, W. V. A dose threshold for a medium transfer bystander effect for a human skin cell line. Radiat. Res. 2006, $166,19-23$. 